\title{
Tracing the origin of low diffusivity and CR bubbles around sources
}

\section{Benedikt Schroer, ${ }^{a, b, *}$ Oreste Pezzi, ${ }^{a, b, c}$ Damiano Caprioli, ${ }^{d, e}$ Colby Haggerty ${ }^{d, f}$ and Pasquale Blasi $^{a, b}$}

${ }^{a}$ Gran Sasso Science Institute,

Viale F. Crispi 7, 67100 L'Aquila, Italy

${ }^{b}$ INFN/Laboratori Nazionali del Gran Sasso,

Via G. Acitelli 22, Assergi (AQ), Italy

${ }^{c}$ Istituto per la Scienza e Tecnologia dei Plasmi, Consiglio Nazionale delle Ricerche, Via Amendola 122/D, I-70126 Bari, Italy

${ }^{d}$ Department of Astronomy and Astrophysics, The University of Chicago,

5640 S Ellis Avenue, Chicago, IL 60637, USA

${ }^{e}$ Enrico Fermi Institute, The University of Chicago,

5640 S Ellis Avenue, Chicago, IL 60637, USA

${ }^{f}$ Institute for Astronomy, University of Hawaii, 2680 Woodlawn Drive, Honolulu, HI 96822, USA

E-mail: benedikt.schroer@gssi.it

Cosmic rays leave their sources mainly along the local magnetic field present in the region around the source and in doing so they excite both resonant and non-resonant modes through streaming instabilities. The excitation of these modes leads to enhanced particle scattering and in turn to a large pressure gradient that causes the formation of expanding bubbles of gas and self-generated magnetic fields. By means of hybrid particle-in-cell simulations, we demonstrate that, by exciting this instability, cosmic rays excavate a cavity around their source where the diffusivity is strongly suppressed. This phenomenon is general and is expected to occur around any sufficiently powerful cosmic ray source in the Galaxy. Our results are consistent with recent $\gamma$-ray observations where emission from the region around supernova remnants and stellar clusters have been used to infer that the diffusion coefficient around these sources is $\sim 10-100$ times smaller than the typical Galactic one.

$37^{\text {th }}$ International Cosmic Ray Conference (ICRC 2021)

July 12th - 23rd, 2021

Online - Berlin, Germany

\footnotetext{
*Presenter
} 


\section{Introduction}

With new $\gamma$-ray and $x$-ray observations over the past years there was a big focus on understanding and studying the sources of CRs. These observations confirmed the standard theoretical picture in which CRs are accelerated at supernova remnant (SNR) shocks via diffusive shock acceleration. In this picture the accelerated particles form a precursor in front of the shock of a typical size of the order of the radius of the remnant itself $(\sim \mathrm{pc})$. The size of this precursor determines the maximum energy of the particles and is often put by hand in the form of a free escape boundary at a given distance from the shock. Once the diffusion length is comparable to this size particles will escape into the surrounding ISM and once their density drops below the Galactic average CR density we call them CRs. While the main essence of this picture, i.e. the acceleration at the shock, found confirmation in observations, there was little focus on understanding the transition of these accelerated particles into CRs in the ISM.

In the last decade the amount of puzzling observations from circumsource regions where this transition is expected to occur increased. Independent of whether the CR source is a stellar cluster [1], a pulsar wind nebula [2] or a SNR [3] it is inferred from $\gamma$-ray observations that the particles' diffusion coefficient in regions around the source on scales $10-100 \mathrm{pc}$ has to be $\sim 100$ times smaller than the Galactic average diffusion coefficient inferred by secondary-to-primary ratios.

In this work, as discussed also in [4], we provide a possible explanation for this suppression for a typical SNR while similar considerations might apply to other CR sources as well. There have been similar attempts to explain the low diffusivity around sources, however all of them focused on the excitation of the resonant streaming instability and arrived at the conclusion that the achieved suppression is not enough to explain observations at high energies [5-8]. The novelty in this work is that we show that accelerated particles are able to excite the non-resonant streaming instability [NRSI; 9, 10] after leaving the source. This instability has a larger growth rate than the resonant one, therefore possibly overcoming the limiting factors of the resonant instability. Opposite to the standard picture in which this instability is invoked only at the shock and in the CR precursor, we show that the condition for its excitation is fulfilled also by the particles that left the source and they are able to confine themselves in a large circumsource region. The calculation is performed similar to previous attempts in the flux tube assumption: particles escape the SNR along the local magnetic field line and fill a one dimensional flux tube. Since diffusion perpendicular to the local magnetic field is highly suppressed, the tranverse size of the flux tube is expected to coincide with the extension of the source. We study this effect first with an analytical estimate to demonstrate that the condition for the excitation of the NRSI are fulfilled for a typical SNR and make predictions about the evolution of the system which are then tested via two dimensional hybrid particle-in-cell simulations [11].

Furthermore we find another important effect which was neglected in all previous literature on the topic, i.e., the flux tube of escaping particles becomes highly overpressurized because particles are trapped near the source. The resulting pressure gradient with respect to outside the tube leads to a transverse expansion, which invalidates the underlying assumption of a one dimensional flux tube of both our analytical estimate and previous attempts to explain the reduced diffusion coefficient in these regions.

In Sect. 2, we first introduce our analytical estimate of the relevant quantities leading to the onset 
of the instability and its implications. Then, in Sect. 3, we introduce the kinetic simulations used to model the motion of CRs in the ISM after they have left the sources. Our main results and their implications are discussed in Sect. 4, while we conclude in Sect. 5.

\section{Escaping particles}

As discussed above in the standard picture of CR transport particles are accelerated at a SNR shock and the highest-energy particles escape into the ISM because their diffusion length becomes larger than the precursor. At any given time t during the Sedov-Taylor phase there is a flux of high energy particles leaking into the ISM, the flux of these particles can be demonstrated to be the same as at the shock [12]. Once in the ISM they will start diffusing due to scattering on the preexisting ISM magnetic turbulence and this is described by the Galactic diffusion coefficient, which is of the order of $D(E) \simeq 3 \times 10^{28} E_{\mathrm{GeV}}^{1 / 2}$, for $E \gtrsim 10 \mathrm{GeV} / \mathrm{n}$. While more refined treatments of Galactic diffusive transport return better descriptions of its normalisation and energy dependence [e.g., 13, 14], the simplified parametrization chosen here keeps the presented argument simple while not affecting the main conclusions reached later. The diffusion coefficient is related to the diffusion length $\lambda$ by $D(E)=v \lambda(E) / 3$. Assuming $v \approx c$ ( $c$ being the speed of light), one obtains $\lambda(E) \simeq 1 \mathrm{pc} E_{\mathrm{GeV}}^{1 / 2}$. To determine if particles diffuse around the source it is important to compare this scale to the coherence length of the Galactic magnetic field $l_{c}$ which is normally taken to be of the order of $50 \mathrm{pc}$. It follows that particles above $2.5 \mathrm{TeV}$ do not diffuse on scales of $50 \mathrm{pc}$ around the source but rather follow the local magnetic field line. Due to their gyration they are expected to fill a flux tube of vertical extent equal to the size of the source as depicted in Fig. 1 with a number density of $n_{C R}(>E) \approx\left(\frac{E_{S}}{E}\right) \frac{\xi_{C R}}{\pi R_{S}^{2} c T_{S} \Lambda}$, where we assumed that the differential spectrum of particles is $\sim E^{-2}$ and $\Lambda=\ln \left(E_{\text {max }} / m c^{2}\right) \sim 15$. The energy released in the form of kinetic energy of the expanding gas is assumed to be $E_{s}=10^{51} \mathrm{erg}$ and $10 \%$ of this energy is converted into CRs, consistent with typical estimates for SNRs [15]. The energy is released over a typical time scale $T_{S}$ which we choose to be of the order of the beginning of the Sedov-Taylor phase ( $\sim 300 \mathrm{yr})$ and $R_{S}$ is the corresponding radius of the SNR $(\sim 3 \mathrm{pc})$.

The CR energy density can then easily be estimated to be $n_{C R}(>E) E \sim 54 \mathrm{eV} \mathrm{cm}^{-3}$, which substantially exceeds the energy density of the typical Galactic magnetic field which is $\sim 0.2 \mathrm{eV} \mathrm{cm}^{-3}$ for $B_{0}=3 \mu \mathrm{G}$. It is easy to check that the CR energy density changes only by a factor of 2 at $2.5 \mathrm{TeV}$ energies even when assuming observationally-preferred [13] and theoretically-motivated [16, 17] steeper spectra, e.g., $E^{-2.3}$, and therefore remains much larger than the energy density of the Galactic magnetic field even in more conservative cases. This holds true also for the low-energy CRs released after the end of the Sedov-Taylor phase which propagate diffusively in the source surroundings. In Ref. [10] it was shown that a NRSI is excited if the following condition is fulfilled:

$$
n_{C R}\left(E>E_{0}\right) E \frac{v_{D}}{c} \gg \frac{B_{0}^{2}}{4 \pi},
$$

with $v_{D}$ the drift velocity of CRs, in this case $\sim c$ and $B_{0} \approx 3 \mu \mathrm{G}$ the magnetic field strength. Hence, since we showed that the energy density of CRs $n_{C R}\left(E>E_{0}\right) E$ exceeds the energy density of the magnetic field, the instability is excited inside the flux tube. Note that the condition in Eq. 1 depends only on the flux of escaping particles $n_{C R}\left(E>E_{0}\right) v_{D}$. Since the excitation of the NRSI 


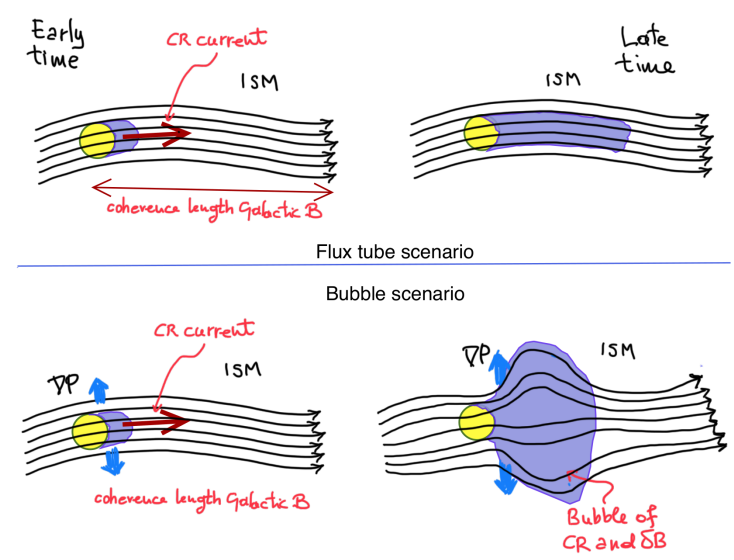

Figure 1: Sketch depicting the main difference between the flux tube scenario and the bubble escape scenario.

is often invoked at SNR shocks to increase the maximum achievable energy, it is not surprising that the condition holds in the tube as well because we do not expect drastically larger magnetic fields in the ISM compared to at the shock and the fluxes are the same.

The fastest-growing mode grows with a growth rate $\gamma_{\max }=k_{\max } v_{A}$ on length scales of $k_{\max }^{-1}$ where $k_{\text {max }} B_{0}=\frac{4 \pi}{c} J_{C R}(>E)$, the Alfvén speed $v_{A}=B_{0} / \sqrt{4 \pi m_{i} n_{0}}$, with $n_{0}$ the number density of the ISM and $m_{i}$ the ion mass and the electric current of particles with energy $\gtrsim E: J_{C R}(>$ $E)=e n_{C R}(>E) c$. Due to $k_{\text {max }}^{-1}$ being much smaller than the gyroradius of the particles driving the current, the current remains unaffected by the growth of the instability in the beginning and the magnetic field keeps growing on time scales of $\gamma_{\max }^{-1} \approx 1.1(\mathrm{E} / 2.5 \mathrm{TeV}) \mathrm{yr}$ until it eventually saturates. The magnetic field is expected to saturate once the energy density of the amplified field and the one of the escaping particles become comparable, which was shown to be equivalent to assuming that power is transferred from small scales $k_{\max }^{-1}$ to the scale of the particles' gyroradius in the amplified magnetic field [10,18]. This condition combined with the above parameters gives an estimate for the saturated $\delta B / B_{0}$ of a few. As explained above at saturation this field is expected to be turbulent on scales of the gyroradius of the particles in the current which for $2.5 \mathrm{TeV}$ particles corresponds to $\sim$ few $10^{-4} \mathrm{pc}$, much smaller than $l_{c}$. Hence, even though the magnetic field strength does not change by a lot, the effects on particle diffusion are dramatic because the turbulent component of the field changes significantly. In the above case with a spectrum of $E^{-2}$ we expect Bohm diffusion after saturation [e.g., 19, 20], which gives a diffusion coefficient of $D(E)=r_{L} c / 3 \approx 10^{25} \mathrm{~cm}^{2} \mathrm{~s}^{-1}$ at $2.5 \mathrm{TeV}$ energies, which is several orders of magnitude smaller than expected at the same energy for Galactic CRs. Therefore, CRs escaping their sources into the ISM generate magnetic instabilities which self confine them in the circumsource region. This effect was previously studied only for the resonant streaming instability [6-8,21], where its effectiveness was limited by damping processes. However, the extension to the NRSI could overcome this limitation due to its higher growth rate. The NRSI keeps growing even after the transition from ballistic escape to diffusion because Eq. 1 depends only on the flux of escaping particles which stays the same in the two transport regimes. Therefore, the cosmic ray density $n_{C R}$ has to increase significantly as the drift velocity $v_{D}$ reduces due to the transition. As a consequence the pressure of escaping particles 
inside the tube increases drastically and due to the isotropization of the diffusing particles a strong pressure gradient along the perpendicular directions builds up. The above estimates show that the energy density and hence the pressure of the escaping particles dominates over the background energy density even when the particles escape ballistically. Hence, once particles start diffusing the pressure in the tube is completely dominated by the escaping particles. This large overpressure with respect to the ambient medium will ultimately lead to an expansion of the tube in the transverse direction in an attempt to reach pressure equilibrium which was so far neglected in the literature. This expansion is expected to continue until either pressure balance is achieved or damping of the turbulence allows faster particle escape, which if nothing peculiar happens should again lead to the excitation of the nonresonant instability and the effects described above. The final size of the CR bubble can be estimated for the first case to be roughly $L \approx\left(\xi_{C R} E_{S} / \Lambda P_{I S M}\right)^{1 / 3} \sim 60 \mathrm{pc}$, where $P_{I S M}$ is the pressure of the ISM. This expected size compares well with the size of the regions of reduced CR diffusivity based on $\gamma$-ray observations.

\section{PIC simulations}

For studying the effects of particle self-confinement in the circumsource region we perform 2D and 3D simulations with dHybridR a relativistic hybrid code with kinetic ions and (massless, charge-neutralizing) fluid electrons [11,22]. Hybrid codes are better suited to self-consistently simulate the long-term, large-scale coupling of CRs and background plasma than fully-kinetic PIC codes as they do not need to resolve small electron scales, which are usually dynamically negligible. Physical quantities like lengths, time, velocities, number densities and magnetic fields in our simulations are normalized to the ion inertial length $d_{i}=v_{A} / \Omega_{c i}$, the inverse ion cyclotron frequency $\Omega_{c i}^{-1}, v_{A}$, the number density $\left(n_{0}\right)$ and the magnetic field strength $\left(B_{0}\right)$ of the initial background plasma respectively. The temperature of the background ions is chosen such that the thermal ions gyroradius $r_{g, i}=d_{i}$, i.e. $\beta_{i}=2 v_{t h, i}^{2} / v_{A}^{2}=2$. In all simulations all three velocity and field components are retained. The simulation is discretized on a grid of size $5000 \times 7000 d_{i}^{2}$ $\left(1200 \times 1200 \times 1200 d_{i}^{3}\right)$, with $7500 \times 10500$ cells $(1440 \times 1440 \times 1440)$ in $2 \mathrm{D}(3 \mathrm{D})$. For CRs we impose open boundary conditions in each direction while for the plasma the conditions are open in the $x$-direction and periodic in the remaining directions. The simulaton box is filled with a background magnetic field along the $x$-direction with a strength $B_{0}$. The background plasma is initialized with $N_{\mathrm{ppc}}=4$ particles per cell, following a Maxwellian with density $n_{0}$, while CRs are injected with $N_{\mathrm{ppc}}=16$ at the $x=0$ boundary between $3200 d_{i}<y<3800 d_{i}$ isotropically with momentum $p_{\text {total }}=100 m v_{A}$, i.e. Lorentz factor $\gamma \approx 5$ and $n_{\mathrm{CR}}=0.0133 n_{0}$ to mimic the escaping particles from a finite sized source. In our 3D simulation CRs are injected in the yz-plane in a circle of radius $80 d_{i}$ in the middle of the plane with a density $n_{C R}=0.01$ as a cold beam along x. CR injection is continuous in time and numerical parameters are chosen to reproduce the ratio of energy densities of CR particles and thermal energy of the SNR case above, i.e. $\sim\left(n_{\mathrm{CR}} / n_{0}\right)\left(c / v_{A}\right)^{2} \gamma \sim 26$ in $2 \mathrm{D}$ and $\sim 125$ in $3 \mathrm{D}$.

\section{Results}

The excitation of streaming instability acts as a bootstrapping process for seeding the overpressurized region around the source. Once the escaping particles start scattering on the self-generated 

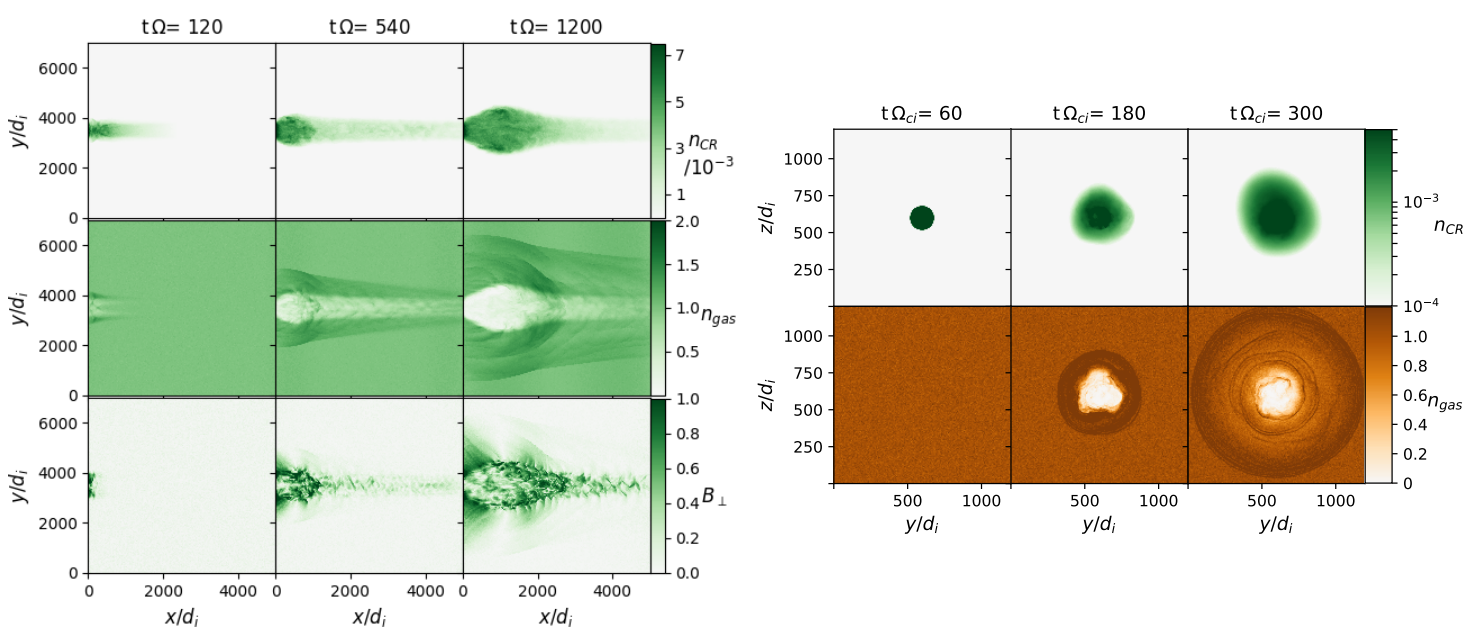

Figure 2: Left panel: CR density and background gas density and magnetic field of the 2D simulation in the xy-plane at three different times. Right panel: CR density and background gas density of the 3D simulation in the yz-plane averaged along the $\mathrm{x}$-axis at three different times.

magnetic turbulence, their bulk velocity along the $x$-direction decreases and as described above their number density increases. This effect and the subsequent formation of a CR bubble can be seen both in our 2D and 3D simulations see Fig. 2 which shows quantities such as CR density, background plasma density and perpendicular magnetic field at an early, intermediate and late time in the simulation in order to illustrate their temporal evolution. Initially CRs escape along the background magnetic field as expected, hence filling a flux tube of the transverse size of the source itself as can be seen in the left image of the top row of Fig. 2. Due to the high particle flux magnetic instabilities are excited, slowing down the particles and trapping them close to the injection region. As a consequence an overpressurized bubble of CRs forms at intermediate times in Fig. 2. In the second row of Fig. 2 one can see that the background plasma gets expelled out of this region, forms an envelope of increased density around the bubble and launches density waves outward. When these waves hit the periodic boundaries of the background plasma, we stop our simulations. As a consequence of the large overpressure the bubble itself starts expanding as well in all directions as can be seen at large times in Fig. 2. The transverse expansion is evident in the figure and renders the flux tube assumption invalid for these parameters. Comparing the left and right plot of Fig. 2 it is clear that the phenomenon appears both in $2 \mathrm{D}$ and $3 \mathrm{D}$ and is therefore not a relic of the reduced dimensionality of the 2D simulation. The observed formation and expansion of the bubble line up very well with the expectations based on simple analytical arguments presented in the first part of this article. The only difference between our 2D and 3D simulations is that we see more background gas mixing inside the bubble, i.e. the evacuation has not such a sharp boundary as in the 2D case.

\section{Conclusions}

In this article we investigated the escape of CRs from their source. Once CRs leave the source environment and enter into the ISM we demonstrated that the flux of escaping particles is sufficient 
to excite the NRSI. Since the condition for its excitation depends only on the particle flux and the magnetic field, it is easy to understand that the NRSI is excited in the flux tube of escaping particles as long as the magnetic field at the shock and in the tube are of the same order of magnitude, because the flux can be shown to be the same. This inhibits the ballistic escape of TeV CRs around their sources and forces them to start diffusing on much smaller scales than expected by the mean Galactic diffusion coefficient. As a consequence, a large pressure gradient with respect to the ambient ISM forms which results in the expansion of the CR flux tube in the transverse direction. This overpressurized CR bubble leads to a partial evacuation of the region.

Based on our analytical estimates and our simulations we expect that all typical CR sources should be surrounded by extended regions of reduced diffusivity and local overdensity of CR particles. The finite box size of our simulations does not allow us to follow the bubbles evolution until its expansion stops. However, it seems reasonable to expect that the bubble keeps expanding until pressure equilibrium with respect to the outside ISM is achieved. As presented above, the final size of $\sim 60 \mathrm{pc}$ compares well with the observations of $\gamma$-rays around SNRs [3] and stellar clusters [1]. The existence of these bubbles has many important implications which will be studied in future works. First, due to the enhanced particle trapping close to the source environment CRs can accumulate a significant fraction of their grammage in the source environment. On the other hand, our simulations show the excavation of the background plasma which effectively will counterplay this effect. Without this excavation and a diffusion coefficient reduced by a factor of $\sim 100$ the source grammage alone would be comparable to the one inferred by observations. Second, the diffusion coefficient of electrons will be reduced as well in the bubble region. Because of their higher energy losses, the escape time might actually become comparable to the loss time leading to a possibly steeper spectrum of electrons and an energy cutoff related to the stronger losses.

\section{Acknowledgments}

Simulations were performed on computational resources provided by the University of Chicago Research Computing Center, the NASA High-End Computing Program through the NASA Advanced Supercomputing Division at Ames Research Center, and XSEDE TACC (TG-AST180008). DC was partially supported by NASA (grants NNX17AG30G, 80NSSC18K1218, 80NSSC20K1273, and 80NSSC18K1726) and by NSF (grants AST-1714658, AST-2009326, AST-1909778, PHY1748958, and PHY-2010240).

\section{References}

[1] Aharonian, F., Yang, R., \& de Oña Wilhelmi, E. 2019, Nature Astronomy, 3, 561, doi: 10. 1038/s41550-019-0724-0

[2] Abeysekara, A. U., \& et al. 2017, Science, 358, 911, doi: 10.1126/science . aan 4880

[3] Hanabata, Y., Katagiri, H., Hewitt, J. W., et al. 2014, The Astrophysical Journal, 786, 145, doi: $10.1088 / 0004-637 \mathrm{X} / 786 / 2 / 145$

[4] Schroer, B., Pezzi, O., Caprioli, C., et al. 2021, The Astrophysical Journal Letters, 914, L13, doi: $10.3847 / 2041-8213 /$ ac02cd 
[5] D’Angelo, M., Blasi, P., \& Amato, E. 2016, Phys.Rev.D, 94, 083003, doi: 10 . 1103/PhysRevD . 94.083003

[6] Malkov, M. A., Diamond, P. H., Sagdeev, R. Z., Aharonian, F. A., \& Moskalenko, I. V. 2013, Ap.J., 768, 73, doi: 10.1088/0004-637X/768/1/73

[7] Nava, L., Gabici, S., Marcowith, A., Morlino, G., \& Ptuskin, V. S. 2016, Monthly Notices of the Royal Astronomical Society, 461, 3552, doi: 10. 1093/mnras/stw1592

[8] Nava, L., Recchia, S., Gabici, S., et al. 2019, Monthly Notices of the Royal Astronomical Society, 484, 2684, doi: 10.1093/mnras/stz137

[9] Amato, E., \& Blasi, P. 2009, Monthly Notices of the Royal Astronomical Society, 392, 1591, doi: $10.1111 / \mathrm{j} .1365-2966.2008 .14200 . \mathrm{x}$

[10] Bell, A. R. 2004, Monthly Notices of the Royal Astronomical Society, 353, 550, doi: 10. 1111/j.1365-2966.2004.08097.x

[11] Haggerty, C. C., \& Caprioli, D. 2019, The Astrophysical Journal, 887, 165, doi: 10 . $3847 /$ $1538-4357 / \mathrm{ab} 58 \mathrm{c} 8$

[12] Bell, A. R., Schure, K. M., Reville, B., \& Giacinti, G. 2013, Monthly Notices of the Royal Astronomical Society, 431, 415, doi: 10.1093/mnras/stt179

[13] Evoli, C., Aloisio, R., \& Blasi, P. 2019, Phys.Rev.D,, 99, 103023, doi: 10.1103/PhysRevD. 99.103023

[14] Evoli, C., Morlino, G., Blasi, P., \& Aloisio, R. 2020, Phys.Rev.D,, 101, 023013, doi: 10. 1103/PhysRevD. 101.023013

[15] Caprioli, D., \& Spitkovsky, A. 2014, The Astrophysical Journal, 783, 91, doi: 10.1088/ $0004-637 \mathrm{X} / 783 / 2 / 91$

[16] Caprioli, D., Haggerty, C. C., \& Blasi, P. 2020, The Astrophysical Journal, 905, 2, doi: 10. 3847/1538-4357/abbe05

[17] Haggerty, C. C. \& Caprioli, D. 2020, The Astrophysical Journal, 905, 1, doi: 10.3847/ $1538-4357 /$ abbe06

[18] Caprioli, D., \& Spitkovsky, A. 2014, The Astrophysical Journal, 794, 46, doi: 10.1088/ 0004-637X/794/1/46

[19] —. 2014, The Astrophysical Journal, 794, 47, doi: 10 . 1088/0004-637X/794/1/47

[20] Reville, B., \& Bell, A. R. 2013, Monthly Notices of the Royal Astronomical Society, 430, 2873, doi: $10.1093 / \mathrm{mnras} / \mathrm{stt} 100$

[21] Blasi, P., Amato, E., \& D’Angelo, M. 2015, Physical Review Letters, 115, 121101, doi: 10. 1103/PhysRevLett. 115.121101

[22] Gargaté, L., Bingham, R., Fonseca, R. A., \& Silva, L. O. 2007, Computer Physics Communications, 176, 419, doi: 10.1016/j.cpc.2006.11.013 